\title{
Ultralong Organic Room-Temperature Phosphorescence of Electron-donating and Commercially Available Host and Guest Molecules through Efficient Förster Resonance Energy Transfer
}

\author{
Yeling Ning, ${ }^{[a]}$ Junfang Yang, ${ }^{[b]}$ Han Si, ${ }^{[a]}$ Haozhong Wu, ${ }^{[a]}$ Anjun Qin, ${ }^{*[a]}$ Xiaoyan Zheng ${ }^{*[b]}$ and Ben \\ Zhong Tang ${ }^{[a, c]}$ \\ [a] Y. Ning, H. Si, H. Wu, Prof. A. Qin, Prof. B. Z. Tang \\ State Key Laboratory of Luminescent Materials and Devices, Guangdong Provincial Key Laboratory of Luminescence from Molecular Aggregates, SCUT- \\ HKUST Joint Research Institute, AIE Institute, Center for Aggregation-Induced Emission, South China University of Technology (SCUT) \\ Guangzhou 510640, China \\ E-mail: msqinaj@scut.edu.cn \\ [b] J. Yang, Prof. X. Zheng \\ Beijing Key Laboratory of Photoelectronic/Electrophotonic Conversion Materials, Key Laboratory of Cluster Science of Ministry of Education, Beijing \\ Institute of Technology \\ Beijing 100081, China \\ E-mail: xiaoyanzheng@bit.edu.cn \\ [c] Prof. B. Z. Tang \\ Department of Chemistry, Hong Kong Branch of Chinese National Engineering Research Centre for Tissue Restoration and Reconstruction, Institute for \\ Advanced Study, and Department of Chemical and Biological Engineering, The Hong Kong University of Science \& Technology (HKUST) \\ Clear Water Bay, Kowloon, Hong Kong, China
}

\begin{abstract}
Ultralong organic room-temperature phosphorescence (RTP) materials have attracted great attention recently due to its diverse application potentials. Several ultralong organic RTP materials mimicking the host-guest architecture of inorganic systems have been exploited successfully. However, complicated synthesis and high expenditure are still inevitable in these cases. Herein, we develop a series of novel host-guest organic phosphore systems, in which all chromophores are electron-rich, commercially available and halogen atom free. The maximum phosphorescence efficiency and the longest lifetime reach at $23.6 \%$ and $362 \mathrm{~ms}$, respectively. Most importantly, experimental results and theoretical calculation indicate that the host molecules not only play a vital role in providing a rigid environment to suppress non-radiative decay of the guest, but also show a synergistic effect to the guest through Förster energy transfer (FERT). The commercial availability, facile preparation and unique properties also make these new host-guest materials an excellent candidate for anti-counterfeiting devices.
\end{abstract}

Room-temperature phosphorescence (RTP) materials have arisen considerable attention because of their distinctive photophysical properties such as long emission lifetimes and the excited state energy, which can be widely applied in data encryption, anti-counterfeiting, background-free bioimaging, chemical sensors, and so on. ${ }^{[1]}$ Although nature is abundant in inorganic phosphorescence materials such as luminous pearls and glow-in the dark stones, most of them suffer from some intrinsic problems, including high cost, potential toxicity, and low biocompatibility etc. For example, a highly efficient RTP system of $\mathrm{SrAl}_{2} \mathrm{O}_{4}$ doped with europium and dysprosium was developed in the mid-1990s, and this inorganic system formed the basis of most commercial glow-in-the-dark paints because of its long emission (> $10 \mathrm{~h}$ ) and high durability. ${ }^{[2]}$ However, this system requires not only rare-earth elements for long-lived emission but also very high fabrication temperatures of more than $1000^{\circ} \mathrm{C}$. Moreover, the manufacture of paints from the insoluble $\mathrm{SrAl}_{2} \mathrm{O}_{4}$ requires tedious procedures, including grinding of the compounds into micrometer-scale powders for dispersion into solvents or matrices, and light scattering by the powders prevents the formation of transparent paints. ${ }^{[3]}$ It is urgent to explore another counterpart with extraordinary features.

Inspired by generally accepted principle in inorganic RTP systems that impurity is responsible and involved three steps: (1) excitation of the guest species (impurity), (2) trapping of the excited electrons by defects in host matrices, and (3) slow charge recombination of the trapped electrons by thermal energy, ${ }^{[4]}$ the researchers have successfully developed some ultralong organic phosphorescence materials imitating the host-guest architecture of minerals. ${ }^{[5]}$ However, complicated synthesis and high expenditure are still inevitable in these systems. Moreover, electron-withdrawing species is necessary. For example, recently, Adachi et al. obtained a purely organic afterglow lasting for more than $1 \mathrm{~h}$ via blending one electron-donating guest in an electronwithdrawing host, namely D-A structure, but the overall emission quantum yield was only $7 \%$ in air. ${ }^{[6]}$ Tang et al. developed a series of purely organic host-guest materials which are both electrondeficient, and halogen atoms, carbonyl groups and cyano units were incorporated. ${ }^{[7]}$ Therefore, a new facile and robust hostguest strategy utilizing only electron-rich materials is proposed.

Our group accidently observed a notable RTP phenomenon in a normal experiment by mixing $N, N, N, N$-tetraphenylbenzidine (TPB), triphenylamine (TPA) and triphenylphosphine (TPP) (Figure 1a). As we all know, TPA and TPP are commonly used starting materials in organic experiments, and TPB is a widely employed hole-transport material in organic light-emitting diodes (OLEDs). ${ }^{[8]}$ In other words, all these three molecules are commercially available with extremely low price. Moreover, both host (TPA or TPP) and guest (TPB) molecules are electron-rich and contain no halogen atoms. When TPB was doped into host molecules, an enhanced phosphorescence quantum yield $\left(\Phi_{\mathrm{P}}\right)$ and extended emission lifetime were witnessed. The maximum $\Phi_{\mathrm{P}}$ and the longest lifetime could reach at $23.6 \%$ and $362 \mathrm{~ms}$, respectively. Furthermore, the experimental results and theoretical calculation revealed that the host molecules not only play a vital role in providing a rigid environment and suppressing 

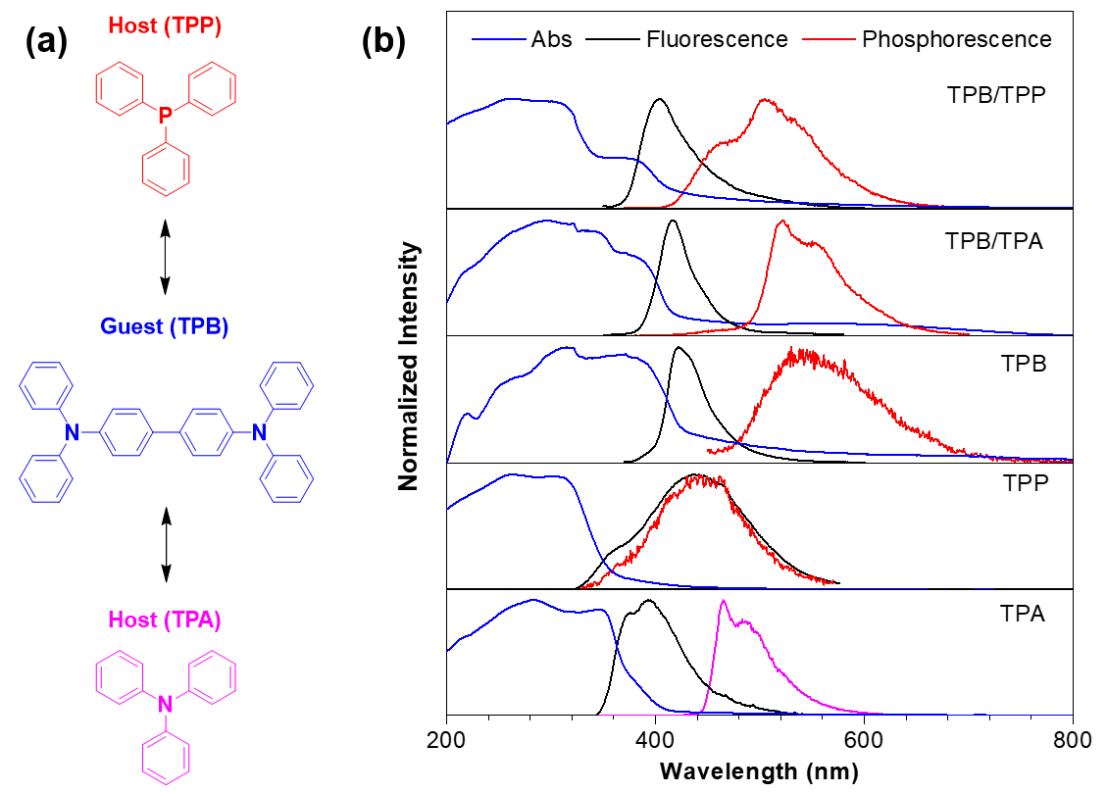

Figure 1. a) The chemical structures of host and guest molecules. b) Absorption, PL and phosphorescence spectra of TPA, TPP, TPB, 1.0 mol \% TPB/TPA and $1.0 \mathrm{~mol} \%$ TPB/TPP crystalline powders at room temperature. (the phosphorescence spectra of TPA was obtained at 77K in magenta line.)

non-radiative decay of the guest, but also show a synergistic effect to the guest in the photophysical process, where Förster resonance energy transfer (FRET) is a key issue to facilitate the phosphorescence of TPB. At the same time, these new hostguest luminescence systems can be facilely fabricated and potentially be an excellent candidate for anti-counterfeiting devices. Thus, the integrated merits including low cost using the most common compounds, absence of halogen atoms, facile preparation, excellent performances and clear mechanism insights of this new host-guest RTP system with all electron-rich species are expected to attract wide attentions and inspire further innovation among the researchers.

To avoid the possible interference of impurities of the host and guest materials, strict purification procedures including column chromatography and recrystallization for three times were used, and their purity was unambiguously confirmed by HPLC measurement (Figure S1). Afterward, the luminescent properties of the individual component and doped materials were investigated at room temperature (Figure $1 \mathrm{~b}$ ), respectively. TPB in the crystalline state exhibits a violet blue fluorescence, emitting at $422 \mathrm{~nm}$ with a lifetime of $1.3 \mathrm{~ns}$ (Table 1 and Figure S2). At the same time, a very weak phosphorescence at $530 \mathrm{~nm}$ and a lifetime of only $9.7 \mu$ s under ambient condition were recorded. The large twisted angle of the biphenyl core of TPB was verified to be the origin of RTP (Figure S3a). ${ }^{[9]}$ Meanwhile, strong intermolecular interactions lock the molecular conformation and restrict the molecular vibration, resulting in the reduction of nonradiative transition of triplet excitons to boost RTP under ambient atmosphere (Figure S3b).

Unique luminescence property was also observed on TPP crystalline powders. As shown in Figures $1 \mathrm{~b}$ and S4, an obvious fluorescence emission at $285 \mathrm{~nm}$ was recorded in the photoluminescence (PL) spectrum of TPP in tetrahydrofuran (THF) solution, while no corresponding emission was found in its solid state. Furthermore, the emission peaked at $439 \mathrm{~nm}$ in its crystalline state was supposed to be phosphorescence owing to the long lifetime of $1.4 \mu$ s (Figure S5). Besides the lifetime, this emission peak was further proven by measuring its PL spectrum at $77 \mathrm{~K}$ (Figure S6), from which a similar emission profile was obtained. Different from TPP, TPA only emits fluorescence peaked at $393 \mathrm{~nm}$, but no phosphorescence was recorded at room temperature.

Table 1. Photophysical properties of host, guest molecules and their doped compounds. ${ }^{[a]}$

\begin{tabular}{|c|c|c|c|c|c|c|}
\hline sample & $\begin{array}{l}\Phi_{P L}{ }^{[b]} \\
(\%)\end{array}$ & $\begin{array}{l}\lambda_{\text {em,Fluo }} \\
(\mathrm{nm})\end{array}$ & $\begin{array}{c}\tau \\
(\mathrm{ns})\end{array}$ & $\begin{array}{l}\lambda_{\mathrm{em}, \text { Phos }} \\
(\mathrm{nm})\end{array}$ & $\begin{array}{l}\Phi_{\mathrm{P}} \\
(\%)\end{array}$ & $\begin{array}{c}\tau \\
(\mathrm{ms})\end{array}$ \\
\hline TPB & 22.9 & 422 & 1.3 & 530 & & 0.0097 \\
\hline TPP & 4.6 & $285^{[c]}$ & $0.6^{[\mathrm{d}]}$ & 439 & & 0.0014 \\
\hline TPA & 9.7 & 393 & 2.7 & $465^{[\mathrm{e}]}$ & & \\
\hline TPB/TPP & 45.8 & 404 & 1.4 & 505 & 23.6 & 336 \\
\hline TPB/TPA & 41.6 & 417 & 1.7 & 522 & 19.4 & 198 \\
\hline
\end{tabular}

[a] Guest/Host=1:100 (Molar ratio). [b] Overall quantum yield of samples. [c] Fluorescence emission of TPP in THF. [d] Fluorescence lifetime of TPP in THF. [e] Data was collected at $77 \mathrm{~K}$. 

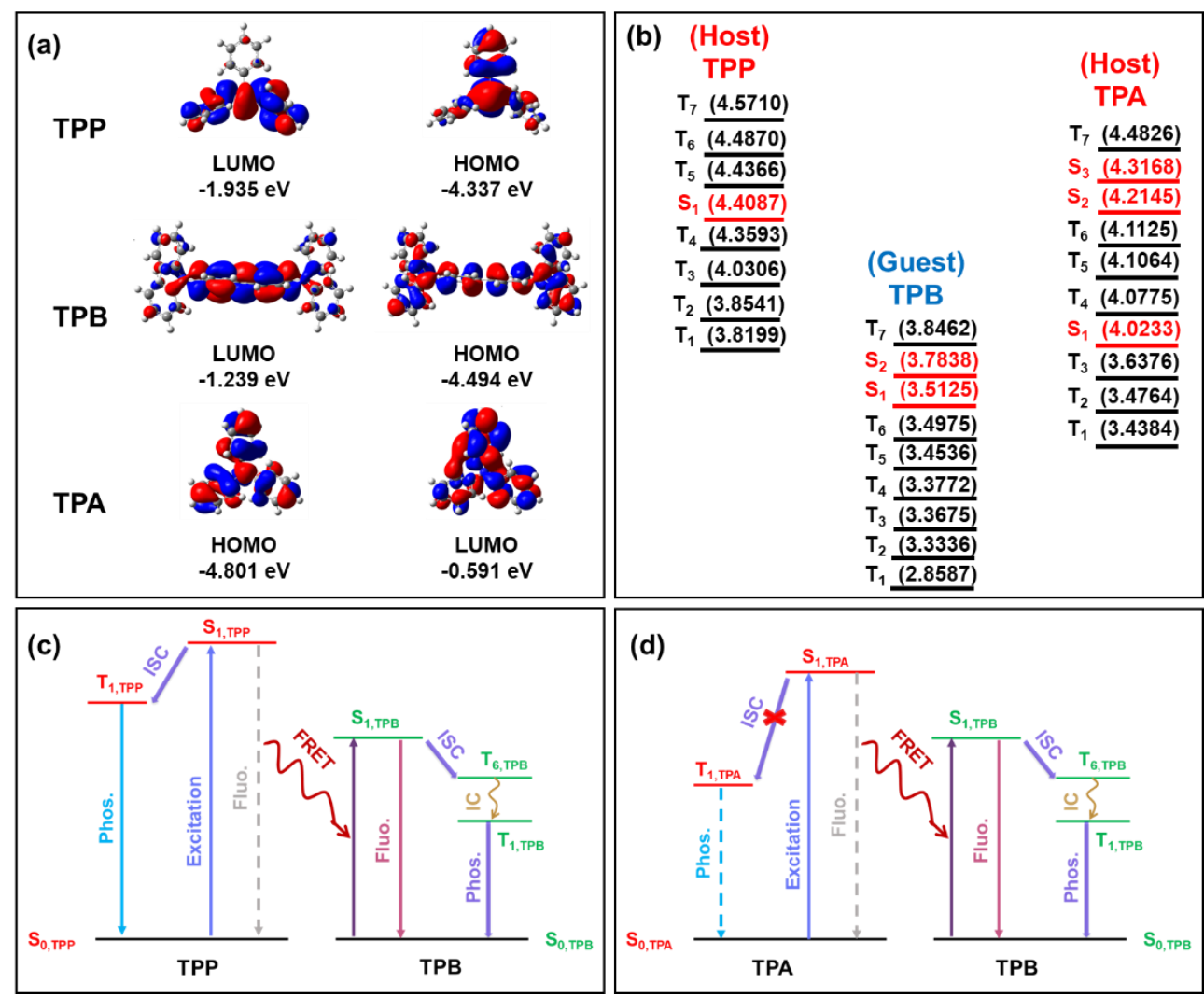

Figure 2. a) Molecular orbitals and the corresponding energies of TPP, TPB and TPA calculated by B3LYP/6-31G(d,p). b) Theoretical energy level diagrams of TPP, TPB and TPA based on crystal data. Schematic diagram of PL processes for c) TPB/TPP and d) TPB/TPA guest-host systems.

The crystals of TPP and TPA showed weak luminescence with overall PL quantum yields $\left(\Phi_{\mathrm{PL}}\right)$ of $4.6 \%$ and $9.7 \%$, respectively. When doping $1.0 \mathrm{~mol} \%$ TPB into TPP or TPA, the formed TPB/TPP and TPB/TPA blends exhibited enhanced quantum yields and ultralong lifetime (Table 1). As can be seen from Figure $1 \mathrm{~b}$, these host-guest systems gave purple fluorescence with the wavelength ranging from 404 to $417 \mathrm{~nm}$. Meanwhile, the maximum phosphorescence of TPB/TPP and TPB/TPA redshifted from 505 to $522 \mathrm{~nm}$, with shoulder peaks at 458 and 540 $\mathrm{nm}$, and $552 \mathrm{~nm}$, respectively. In addition, TPB/TPP and TPB/TPA co-crystals were obtained through careful cultivation, whose $X$-ray diffraction (XRD) patterns exhibited strong and sharp peaks (Figure S7). Furthermore, the formation of co-crystalline structures was confirmed by differential scanning calorimetry (DSC) measurement. The only one melting point of TPB/TPP and TPB/TPA crystalline powders which was different from TPP and TPA themselves (Figure S8), indicating co-crystalline formation of the host and guest molecules. The co-crystals all exhibited high $\Phi_{\mathrm{P}}$ with values of $23.6 \%$ and $19.4 \%$, respectively. According to the phosphorescence decay curves, the RTP lifetime of cocrystals of TPB/TPP and TPB/TPA could reach at 198 to $362 \mathrm{~ms}$ (Figure S9), resulting in a long afterglow that lasts for several seconds under ambient conditions. The relatively stronger phosphorescence $\Phi_{p}$ of TPB/TPP than that of TPB/TPA (Table 1) might be due to the heavy atomic effect of phosphine in the former

In general, the host materials only provide a rigid environment to prevent the triplet excitations from quenching by interaction with oxygen. However, the lifetime of TPB/TPP and TPB/TPA cocrystals at room temperature just enhanced slightly under nitrogen over in air (Figure S10). Therefore, the great luminous ability of host-guest materials under ambient conditions indicates that the rigid host molecules also restrict the motion of the guest ones and decrease the non-radiation transitions, thus improving the light conversion efficiency.

To have a deep insight into aforementioned phenomena, density functional theory calculations were performed to obtain the singlet and triplet energy levels of the host and guest molecules (Figure 2). The results manifested that the spin-orbit coupling constant (SOC, $\xi(\mathrm{S} 1, \mathrm{~T} 1))$ of the TPB was only $0.05 \mathrm{~cm}^{-1}$ due to the lack of heavy atoms, ${ }^{[10]}$ which is consistent with the short lifetime and low quantum yield of the single component of TPB. However, the persistent RTP can be generated by doping TPB into either TPP or TPA host materials. It means that the host and guest molecules must act synergistically in the photo-excited electronic transitions. Thus, the energy levels of TPB, TPP and TPA were calculated. As displayed in Figure 2b, large band gap between the lowest singlet state $\left(\mathrm{S}_{1}\right)$ and lowest triplet state $\left(\mathrm{T}_{1}\right)$ of guest molecules does not facilitate the intersystem crossing (ISC), while the $S_{1}$ of the guest molecule of TPB and the $T_{1}$ of the host molecules of TPP and TPA were very close. By combing the big proportion of spectral overlap between the host emission and the guest absorption (Figure S6), we conclude that the FRET process exists in these host-guest doping systems, in which the TPP or TPA acted as an energy donor and TPB as an acceptor. ${ }^{[11]}$ It is well-known that FRET is a distance-sensitive energy-transfer process and is dependent on distance of the donor and acceptor $\left(R_{\mathrm{DA}}{ }^{6}\right)^{[12,13]}$ 


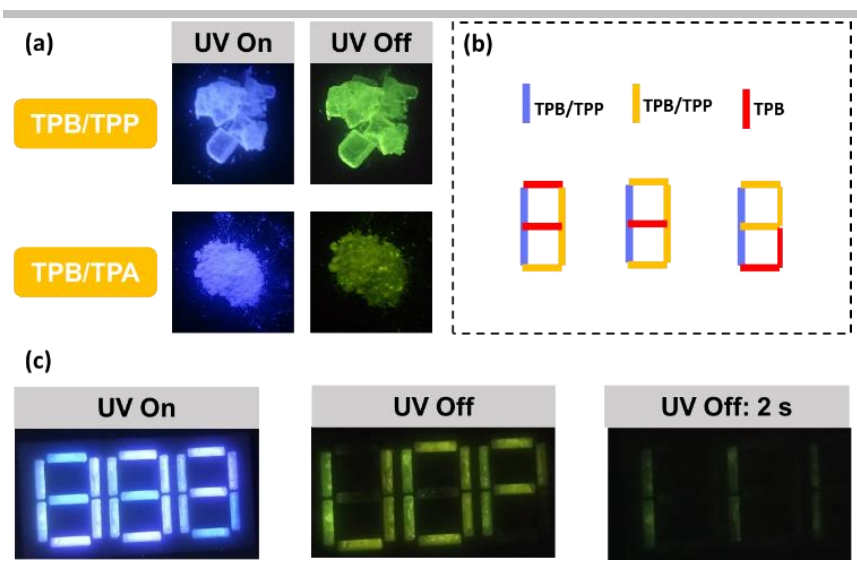

Figure 3. a) $1.0 \mathrm{~mol} \% \mathrm{TPB} / \mathrm{TPP}$ and TPB/TPA crystalline powders before and after the removal of $365 \mathrm{~nm}$ UV irradiation. b) Patterns designed with different phosphors. c) Demonstration of multilevel anti-counterfeiting using the TPB/TPP, TPB/TPA and TPB crystals.

The efficiency of the FRET is usually measured by the energy transfer rate from a host to a guest $\left(k_{\mathrm{ET}}\right)$, the efficiency $\left(\Phi_{\mathrm{ET}}\right)$ and the Förster radius $\left(R_{0}\right)$, at which the FRET is $50 \%$ efficient. The $k_{\mathrm{ET}}$ can be calculated using the following equation: ${ }^{[14]}$

$$
k_{E T}=\frac{1}{\tau_{D}}\left(\frac{R_{0}}{R_{D A}}\right)^{6}
$$

where $\tau_{\mathrm{D}}$ is the decay lifetime of a host in the absence of a guest, $R_{0}$ is the Förster distance, and $R_{\mathrm{DA}}$ is the host-to-guest distance. ${ }^{[12]}$ The efficiency of energy transfer $\Phi_{\mathrm{ET}}$ is given by the following formula:

$$
\Phi_{E T}=\frac{k_{E T}}{k_{E T}+\frac{1}{\tau_{D}}}=\frac{1}{1+\left(\frac{R_{D A}}{R_{0}}\right)^{6}}
$$

The calculated values of $R_{0}, k_{\mathrm{ET}}$ and $\Phi_{\mathrm{ET}}$ of our host-guest systems are listed in Table 2 . It is evident that the $\Phi_{\mathrm{ET}}$ of TPB/TPA system $(91.50 \%)$ is much higher than that of TPB/TPP $(74.33 \%)$. The great distinction of $\Phi_{E T}$ values reveals that TPB/TPA cocrystals experience an absolute FRET process so that no emission originated from TPA appeared in the phosphorescence spectrum. In contrast, TPB/TPP co-crystals underwent an incomplete FRET process following an efficient intersystem crossing from the singlet state of TPP to triplet state, thus causing the extra shoulder peak at $458 \mathrm{~nm}$ originated from the phosphorescence of TPP. Consequently, persistent RTP systems through host-guest strategy involving efficient FRET could well occur in different systems as plotted in Figure 2c and 2d.

Table 2. Förster energy transfer parameters of the host-guest systems. [a]

\begin{tabular}{lllll}
\hline host & guest & $R_{\mathrm{o}}(\mathrm{nm})$ & $K_{\mathrm{ET}}\left(\mathrm{s}^{-1}\right)$ & $\Phi_{\mathrm{ET}}(\%)$ \\
\hline TPP & TPB & 2.60 & $4.83 \times 10^{9}$ & 74.33 \\
TPA & TPB & 3.17 & $3.98 \times 10^{9}$ & 91.50 \\
\hline
\end{tabular}

[a] The detailed computational processes are shown in the Supporting Information.

After having a deep understanding of the mechanism, we demonstrated the potential application of these host-guest RTP systems. Since the guest can be mixed into the hosts at molecular level through facile evaporation process, these co-crystals are of great potential to serve as anti-counterfeiting materials. Considering the similar purple fluorescence color of TPB, TPB/TPP and TPB/TPA crystals, and doped materials all exhibited bright green phosphorescence with various lifetimes of afterglow (Figure 3a), herein, an anti-counterfeiting pattern "888" was fabricated using different luminophores (Figure $3 b$ ). Under a $365 \mathrm{~nm}$ UV-lamp irradiation, the pattern displayed a digital number "888", which became an obvious word "UOP" (which means Ultralong Organic Phosphorescence) visible to the naked eyes due to the synergistic emission of TPB/TPP and TPB/TPA co-crystals after ceasing UV light irradiation instantly. At the end, the digital number of "1 11 " emitting from TPB/TPP appeared after 2 seconds (Figure 3c). Hence, triple data encryption processes have been realized by this simple preparation method, which would be much promising in practical application.

In summary, we present a new strategy to realize purely organic RTP with both high efficiency and ultralong lifetime via mixing commercially available TPB guest into either TPP or TPA host. The experimental results and theoretical simulation indicate that: (1) the rigid construction of host molecules not only play a vital role in avoiding the quenching of the triplet excited states by oxygen, but also restrain the non-radiation transitions to advance the light conversion efficiency; (2) host and guest molecules work synergistically in the photo-excited electronic transition processes; (3) an efficient FRET process is activated in the co-crystals of the host and guest to facilitate the luminescence originated from TPB. This new strategy enjoys the advantages including low cost, absence of halogen atoms, facile preparation and excellent performances, which shows great potentials in practical applications. Therefore, this work broadens the way for the fabrication of purely organic RTP materials and offers a novel platform for the development of outstanding applications.

\section{Acknowledgements}

This work was financially supported by the National Natural Science Foundation of China (21788102 and 21525417), the Natural Science Foundation of Guangdong Province (2019B030301003 and 2016A030312002), and the Innovation and Technology Commission of Hong Kong (ITC-CNERC14S01).

\section{Conflict of interest}

The authors declare no conflict of interest.

Keywords: room-temperature phosphorescence $\cdot$ host-guest system - Förster resonance energy transfer - commercial luminogen $\cdot$ anti-counterfeiting

[1] a) W. Liu, J. Wang, Y. Gong, Q. Liao, Q. Dang, Z. Li, Z. Bo, Angew. Chem. Int. Ed. 2020, 59, 2-8; Angew. Chem. 2020, 132, 2-8; b) Z. He, H. Gao, S. Zhang, S. Zheng, Y. Wang, Z. Zhao, D. Ding, B. Yang, Y. Zhang, W. Yuan, Adv. Mater. 2019, 31, 1807222; c) Y. Su, S. Z. F. Phua, Y. Li, X. Zhou, D. Jana, G. Liu, W. Q. Lim, W. K. Ong, C. Yang, Y. Zhao, Sci. Adv. 2018, 4, 9732; d) K. Zhang, Q. Yu, H. Wei, S. Liu, Q. Zhao, W. Huang, Chem. Rev. 2018, 118, 1770-1839; e) J. Yang, H. Gao, Y. Wang, Y. Yu, Y. Gong, M. Fang, D. Ding, W. Hu, B. Z. Tang, Z. Li, Mater. Chem. Front. 2019, 3, 1391-1397; f) Z. Yang, C. Xu, W. Li, Z. Mao, X. Ge, Q. Huang, H. Deng, J. Zhao, F. L. Gu, Y. Zhang, Z. Chi, Angew. Chem. Int. Ed. 2020, 59, 17451-17455; Angew. Chem. 2020, 132, 17604-17608; g) L. Bian, H. Ma, W. Ye, A. Lv, H. Wang, W. Jia, L. Gu, H. Shi, Z. An, W. Huang, Sci. China Chem. 2020, 63, 1443-1448.

[2] T. Matsuzawa, Y. Aoki, N. Takeuchi, Y. Murayama, J. Electrochem. Soc 1996, 143, 2670-2673. 
[3] Y. Li, M. Gecevicius, J. Qiu, Chem. Soc. Rev. 2016, 45, 2090-2136.

[4] H. Shi, Z. An, Nat. Photonics 2019, 13, 74-75.

[5] a) D. Li, F. Lu, J. Wang, W. Hu, X. Cao, X. Ma, H. Tian, J. Am. Chem. Soc. 2018, 140, 1916-1923; b) J. Wang, Z. Huang, X. Ma, H. Tian, Angew. Chem. Int. Ed. 2020, 59, 1-7; Angew. Chem. 2020, 132, 10014-10019; c) Y. Lei, W. Dai, J. Guan, S. Guo, F. Ren, Y. Zhou, J. Shi, B. Tong, Z. Cai, J. Zheng, Y. Dong, Angew. Chem. Int. Ed. 2020, 59, 16054-16060 Angew. Chem. 2020, 132, 16188-16194.

[6] R. Kabe, C. Adachi, Nature 2017, 550, 384-387.

[7] X. Zhang, L. Du, W. Zhao, Z. Zhao, Y. Xiong, X. He, P. Gao, P. Alam, C. Wang, Z. Li, J. Leng, J. Liu, C. Zhou, J. W. Y. Lam, D. L. Phillips, G. Zhang, B. Z. Tang, Nat. Commun. 2019, 10, 5161.

[8] M. Aonuma, T. Oyamada, H. Sasabe, Appl. Phys. Lett. 2007, 90, 183503.

[9] F. Fries, M. Louis, R. Scholz, M. Gmelch, H. Thomas, A. Haft, S. Reineke, J. Phys. Chem. A 2020, 124, 479-485.

[10] a) S. Hirata, J. Mater. Chem. C 2018, 6, 11785-11794; b) H. Shi, L. Song, H. Ma, C. Sun, K. Huang, A. Lv, W. Ye, H. Wang, S. Cai, W. Yao, Y Zhang, R. Zheng, Z. An, W. Huang, J. Phys. Chem. Lett. 2019, 10, 595600; c) J. Wang, X. Gu, H. Ma, Q. Peng, X. Huang, X. Zheng, S. H. P. Sung, G. Shan, J. W. Y. Lam, Z. Shuai, B. Z. Tang, Nat. Commun. 2018 9, 2963.

[11] H. Wang, B. Yue, Z. Xie, B. Gao, Y. Xu, L. Liu, H. Sun, Y. Ma, Phys. Chem. Chem. Phys. 2013, 15, 35273534.

[12] T. Förster, Ann. Phys. 1948, 2, 55-75.

[13] a) Y. Wang, J. Yang, M. Fang, Y. Yu, B. Zou, L. Wang, Y. Tian, J. Cheng, B. Z. Tang, Z. Li, Matter 2020, 3, 449-463; b) Y. Wang, J. Yang, Y. Gong, M. Fang, Z. Li, B. Z. Tang, SmartMat. 2020, e1006.

[14] T. Förster, Discuss. Faraday Soc. 1959, 27, 7-17. 


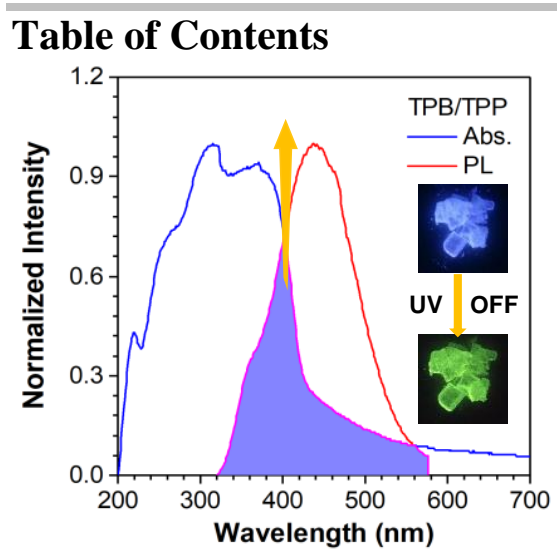

New type of ultralong organic room-temperature phosphorescence (RTP) systems constructed from commercially available host and guest molecules are presented, which show the maximum phosphorescence efficiency and the longest lifetime of $23.6 \%$ and $362 \mathrm{~ms}$, respectively. This new host-guest strategy involving efficient Förster resonance energy transfer (FRET) proves that host molecules not only play passive roles such as rigidifying the guest to suppress the non-radiative decay, but also undergo the excitation and emission processes with guest molecules. 